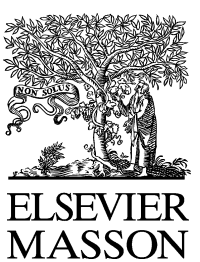

Disponible en ligne sur

Elsevier Masson France

\section{ScienceDirect}

www.sciencedirect.com
EM|consulte

www.em-consulte.com neuropsychiatrie

de l'enfance

et de l'adolescence

Neuropsychiatrie de l'enfance et de l'adolescence xxx (2015) xxx-Xxx

Article original

\title{
Interrompre ou poursuivre la grossesse à l'adolescence : facteurs de risque psychosociaux
}

\author{
Teenage pregnancy and abortion: Psychosocial risk factors \\ A. Greco ${ }^{\text {a, } *}$, J. Reggers ${ }^{\mathrm{b}, 1}$, F. Glowacz ${ }^{\mathrm{a}}$ \\ ${ }^{a}$ Unité de psychologie clinique de la délinquance, des inadaptations sociales et des processus d'insertion, université de Liège, \\ boulevard du Rectorat B33, 4000 Liège, Belgique \\ ${ }^{\mathrm{b}}$ Université de Liège, boulevard du Rectorat B33, 4000 Liège, Belgique
}

\section{Résumé}

Introduction. - Les grossesses à l'adolescence suscitent de nombreuses questions et restent un problème de santé publique au niveau mondial. Elles sont problématiques car elles sont souvent associées à des difficultés socio-économiques, familiales et psychologiques.

Objectif. - L'objectif de cette étude est de comparer les profils psychologiques et sociaux de jeunes filles ayant opté pour une interruption volontaire de grossesse, de celles ayant décidé de la poursuivre, et de jeunes filles n'ayant jamais vécu de grossesse.

Méthode. - L'échantillon se compose de 14 jeunes filles âgées entre 16 et 21 ans dont 7 qui ont décidé de poursuivre leur grossesse et 7 autres de l'interrompre. Ces adolescentes ont été comparées à un groupe témoin de 18 jeunes filles n'ayant jamais été enceintes. Un entretien et des questionnaires structurés leur ont été administrés afin d'évaluer les caractéristiques individuelles et environnementales.

Résultats. - Un milieu familial dépourvu de soutien, des carences affectives, un réseau social restreint, un milieu socio-économique précaire, une mauvaise estime de soi et un désinvestissement scolaire seraient effectivement de puissants prédicteurs de la poursuite d'une grossesse à l'adolescence. Par contre, aucun trait particulier au niveau de la personnalité n'a été démontré.

Conclusion. - Les programmes de prévention doivent, à notre sens, être davantage investis par les politiques de santé publique, sous forme d'informations d'éducation à la sexualité et ce, dès l'enseignement primaire. Toutefois, ces actions doivent dépasser l'information sur la contraception et son utilisation, car la grossesse à l'adolescence est bien plus qu'un défaut ou un échec de contraception, elle répond, pour de nombreuses adolescentes, à des attentes implicites d'une identité nouvelle et de réparation par rapport à des fractures et traumatismes antérieurs. Eu égard aux difficultés psychosociales et au vécu d'adversité des mères adolescentes qui ne sont plus scolarisées, des interventions spécifiques de soutien à la parentalité, en vue de renforcer leurs compétences et sentiments de compétence parentale, et les sensibiliser à leurs propres besoins et à ceux de leur enfant, pourraient être menées à partir d'une clinique en milieu de vie et d'interventions à domicile.

(c) 2015 Publié par Elsevier Masson SAS.

Mots clés : Grossesse à l'adolescence ; Interruption volontaire de grossesse ; Facteurs de risques psychosociaux ; Carences affectives précoces

\begin{abstract} problematic because it is often associated with socio-economic, family and psychological difficulties. not.

\footnotetext{
* Auteur correspondant.

Adressese-mail: antonia.greco@chu.ulg.ac.be (A. Greco), fabienne.glowacz@ulg.ac.be (F. Glowacz).

1 Auteur décédé.
}

Introduction. - Teenage pregnancy arouses lots of questions and remains a worldwide social and public health problem. This phenomenon is

Objective. - Our study aims to identify the individual and environmental factors that influence the teenager's decision whether to keep her child or

Method. - A sample of fourteen girls between 16 and 21 years old was recruited who had been or were pregnant. The girls were divided into 2 groups: seven who decided to continue with their pregnancy and seven others who decided to have an abortion. This group was compared to girls who had never been pregnant $(n=18)$. An interview was carried out and structured questionnaires were administered in order to evaluate individual and environmental characteristics as the age of menarche and the first sexual intercourse, the emotional and sexual education, the social and family 
support, the socio-cultural background and the socio-economic environment. Different other dimensions were analyzed: individual characteristics (Temperament and Character Inventory Junior, Impulsive Behaviour Scale, Coopersmith Self-Esteem Inventory) and family characteristics (Adverse Childhood Experiences) such as childhood family strengths and adverse childhood experiences.

Results and conclusions. - Our study findings showed that a precarious socio-economic environment, a lack of support and affection in the family, emotional deprivations, a restricted social network, low self-esteem and school disinvestments would effectively predict the continuation of a pregnancy. Childhood family strengths seem to be strongly protective against early sexual intercourse, adolescent pregnancy and long-term psychosocial consequences. On the other hand, no particular personality feature was demonstrated. Individual characteristics would thus have less impact on premature pregnancies than environmental characteristics. Prevention programs must stretch beyond the use of contraception, i.e. tend toward an intervention for psycho-socially and socio-economically at risk families, so as to support them in their educational and affective role with their children. Psychological help could also be brought to young girls who have had experiences of maltreatment, to favour self-esteem and raise their awareness of the consequences of sexual risk behaviour. Finally, there should be specific help for the girls during the decision-making period. Counselling during this period should be attentive to the resources and the motivations of the adolescent and to the circumstances of the pregnancy.

(C) 2015 Published by Elsevier Masson SAS.

Keywords: Adolescent pregnancy; Abortion; Psychosocial risk factors; Adverse childhood experiences

\section{Introduction}

À l'heure actuelle, bien que les grossesses à l'adolescence semblent être en légère diminution dans nos sociétés occidentales, elles restent un important problème de santé publique dans beaucoup de pays en voie de développement [1]. Elles représentent $11 \%$ des naissances annuelles dans le monde avec environ 16 millions de jeunes filles âgées entre 15 et 19 ans qui mettent au monde un enfant chaque année. Nonante cinq pour cent de ces naissances ont lieu dans des pays à faible revenu et plus de $50 \%$ en Afrique subsaharienne. Le taux d'interruption volontaire de grossesse est en recrudescence avec une estimation de 4 millions d'avortements chez des jeunes filles âgées entre 15 et 19 ans, ce qui représente environ $10 \%$ de la population mondiale. De plus, 3 millions de ces avortements sont pratiqués dans des conditions à risque [2]. En Europe, c'est au Royaume-Uni que la prévalence des naissances chez les jeunes filles âgées entre 15 et 19 ans est la plus importante avec un nombre de 26 naissances pour mille qui dépasse largement celui de la Belgique (11 pour mille), de la France (11 pour mille), des Pays-Bas (5 pour mille), de l'Italie (7 pour mille), de l'Espagne (13 pour mille) et du Portugal (16 pour mille). En ce qui concerne les interruptions volontaires de grossesse, selon l'OMS, en 2008, 43,8 millions d'avortements ont été pratiqués dans le monde dont $10 \%$ concernent des adolescentes entre 15 et 19 ans. Dans les pays développés, les taux d'interruption volontaire de grossesse chez les adolescentes vont de 0,42\% aux Pays-Bas à 4,57\% aux États-Unis. Les Pays-Bas, l'Espagne, l'Italie, la Grèce, la Belgique et l'Allemagne ont le taux le plus bas. Les États-Unis, la Hongrie, la Nouvelle-Zélande, le Royaume-Uni et le Canada détiennent le taux le plus élevé. Dans les pays sous-développés, il est difficile d'avoir des chiffres exacts car la plupart du temps, les interruptions volontaires de grossesses sont pratiquées de manière illégale. D' ailleurs, selon l'OMS, on estime à 3 millions le nombre d'avortements à risque chez les adolescentes âgées entre 15 et 19 ans [3].

Malgré l'inquiétude que ce phénomène complexe peut susciter, il reste peu abordé dans la littérature francophone. Il est évident que la survenue d'une grossesse fait intervenir de nombreux facteurs. Cependant, ceux qui sont le plus souvent cités dans les études sont le manque de connaissances concernant les moyens de contraception et leur fonctionnement. Selon une étude du Groupe d'action des centres extra-hospitalier pratiquant l'avortement (GACEHPA) de 2006 sur le parcours contraceptif des adolescentes confrontées à une grossesse non désirée, la pilule contraceptive ne serait pas le moyen de contraception le plus adapté aux adolescentes en raison de difficultés dans la prise régulière et de compliance quotidienne déficitaire. Les patchs et les implants seraient plus adaptés mais plus chers et moins bien remboursés que la pilule. Le préservatif, utilisé en début de relation en raison de la crainte de contracter une MST, tend à être moins utilisé lorsque la relation devient plus stable dans le temps [4].

De nombreuses études ont mis en évidence que des vécus d'adversité sur le plan socio-économique, émotionnel et psychoaffectif se retrouvent dans les histoires des jeunes filles qui « tombent enceintes » : parcours de vie difficiles, carences affectives précoces, problèmes socio-économiques, décrochages scolaires, et isolement social [5-10]. Certaines études soulignent les répercussions psychosociales et économiques pour la mère mais également pour l'enfant à venir telles qu'un risque plus élevé de mort foetale, un plus faible poids de naissance, un taux plus élevé de prématuré, des problèmes de développement cognitif et un risque accru d'abus, de négligence ou d'abandon $[6,7]$.

La nature intentionnelle ou accidentelle de la grossesse à l'adolescence est un sujet pris en compte dans la plupart des études sans pour autant arriver à un consensus. La question peut s'avérer effectivement sans grand intérêt, la frontière entre l'intentionnelle et l'accidentelle n'étant plus claire si l'on s'intéresse aux dimensions consciente et inconsciente [11]. Par contre, le questionnement prend toute sa pertinence quand il est étudié au travers du prisme de la contraception, la résistance à la contraception étant considérée comme un des facteurs explicatifs de la grossesse à l'adolescence [12]. Le Van a identifié cinq types de grossesses : la grossesse comme rite d'initiation 
où la jeune fille ne désire pas nécessairement un enfant, elle désire être enceinte. La grossesse SOS où celle-ci n'est pas planifiée mais elle est provoquée par un comportement contraceptif à risque. La grossesse insertion où l'objectif est de combler un vide et d'accéder à un statut social reconnu par l'entourage de la jeune fille. La grossesse identité, quant à elle, n'est pas consciemment planifiée mais elle est le fruit d'un comportement contraceptif à risque. Enfin, la grossesse accidentelle est totalement imprévue et involontaire. La jeune fille, ici, ne pense pas courir le risque de «tomber enceinte». Cette typologie s'appuyant sur la différenciation des mécanismes intentionnels et conscients s'inscrivant dans des dynamiques relationnelles permet de reconnaître diverses fonctions à la grossesse : fonction de réparation, de construction identitaire, d'autonomisation et d'adaptation [9].

Il est néanmoins reconnu que la survenue d'une grossesse est influencée par des facteurs multiples liés à l'environnement socio-économique, démographique et culturel, mais également à des facteurs psychologiques et psychosociaux, la pauvreté et les faibles attentes des jeunes filles concernant leurs perspectives d'avenir sur le plan social et scolaire entrant fortement en ligne de compte. La survenue d'une grossesse dans des conditions socio-économiques défavorables peut alors être vue comme une stratégie d'adaptation pour échapper à un avenir socialement peu valorisant au niveau scolaire et professionnel, la grossesse permettrait ainsi d'accéder à un statut social et donnerait le leurre à la jeune fille de surmonter des carences et traumatismes affectifs antérieurs. Selon Susan Hillis et al. [6], les ressources familiales durant l'enfance protègent fortement des grossesses à l'adolescence, d'une activité sexuelle précoce et des conséquences psychosociales négatives à long terme. Par ressources familiales, on entend proximité familiale, assistance, loyauté, protection, amour et réactivité aux besoins de santé. Par ailleurs, un enfant d'une mère adolescente aurait plus de risques d'avoir des problèmes comportementaux et d'avoir plus d'activités criminelles. Il est bien sûr évident que ces risques ne sont pas liés à la grossesse mais sont surtout associés à plusieurs facteurs qui ont amené la grossesse tels que le manque de supervision et de soutien parental, des abus, de la négligence dans l'enfance et des soins inadéquats [6,7]. Selon Faucher, le statut socio-économique, plus qu'à l'âge de la mère, contribue aux conséquences négatives. Il insiste également sur la diversité interindividuelle et sur l'importance d'un soutien de l'entourage. Les liens familiaux joueraient dès lors un rôle de protection important dans la prévention des conséquences négatives d'une grossesse à l'adolescence et contribueraient à une sexualité sécuritaire [13]. De la même manière, Loignon décrit de l'isolement social, des habitudes de vie précaires, un décrochage scolaire, de la maltraitance, du stress et de la dépression chez les jeunes filles mères [10].

Sur le plan cognitif, le processus de parentalité pourrait être affecté par l'immaturité de l'adolescent dont le développement cognitif est toujours en cours. En effet, l'adolescente aurait du mal à se décentrer, à anticiper et à mettre en perspective ses actions et les conséquences de ses actions, ce qui pourrait rendre difficile l'ajustement à la grossesse et aux besoins de l'enfant. Au niveau identitaire, la parentalité à l'adolescence interfère et intervient au niveau des processus d'individuation et d'autonomie qui sont deux tâches importantes du développement de l'adolescente [14]. D'autre part, certaines études ont démontré des risques accrus de dépression et d'anxiété chez des jeunes mères $[10,15]$. Cependant, il est à noter que la relation existant entre troubles psychiatriques et grossesse à l'adolescence est à prendre avec précaution étant donné que celle-ci s'inscrit, dans la plupart des cas, dans un parcours de vie tumultueux et difficile, jalonné de carences affectives et de traumatismes de toutes formes. En effet, ces jeunes mères présentent très souvent un style d'attachement insécure influençant la qualité de leurs relations affectives [16-18]. À la lumière de tout ce qui vient d'être dit, on constate que le phénomène de grossesse à l'adolescence est complexe et qu'il fait intervenir l'interaction d'un grand nombre de facteurs. Chaque facteur a une part contributive sans pour autant en expliquer totalement la cause.

\section{Objectif de l'étude}

L'objectif de cette étude est comparer les profils psychologiques et sociaux de jeunes filles ayant opté pour une interruption volontaire de grossesse, de celles ayant décidé de la poursuivre, et de jeunes filles n'ayant jamais vécu de grossesse. Nous avons identifié les éléments psychologiques, familiaux et sociaux saillants pouvant intervenir dans la décision de la poursuite ou non d'une grossesse à l'adolescence. Pour ce faire, une approche mixte a été retenue, d'une part, qualitative, à partir d'analyses d'entretiens semi-structurés et, d'autre part, comparative, de dimensions psychologiques et sociales évaluées à partir d'instruments validés.

\section{Méthodologie}

\subsection{Participants}

L'objectif initial était de recruter des jeunes filles dont l'âge était inférieur ou égal à 19 ans, ayant subi une IVG dans l'année et des jeunes filles ayant décidé de mener leur grossesse à terme, enceintes de trois à neuf mois. Toutefois, les difficultés à trouver des sujets répondant aux critères initiaux nous ont amené à élargir les critères d'inclusion. La tranche d'âge a été élargie à 21 ans et ont été incluses des jeunes filles ayant accouché dans l'année écoulée, ces dernières étant en général davantage disposées à parler de leurs expériences, que les jeunes filles ayant récemment découvert leur grossesse qui, peut-être, se trouvent encore dans une période de questionnement et de doute. Un courrier a été adressé à une quarantaine de centres. Ces adolescentes ont été recrutées dans différentes institutions tels que des plannings familiaux, des maisons maternelles et des hôpitaux pratiquant les IVG.

L'échantillon est finalement composé de 32 jeunes filles âgées entre 16 et 21 ans. Il comprend 14 jeunes filles, 7 qui ont décidé de poursuivre leur grossesse (moyenne d'âge de 17 ans et 7 mois) et 7 autres qui ont décidé de l'interrompre (moyenne d'âge de 16 ans et 4 mois) (Tableau 1). Au moment de l'entretien, 6 jeunes filles sur 14 vivaient en institution dont cinq du groupe grossesse 
Tableau 1

Présentation des jeunes filles.

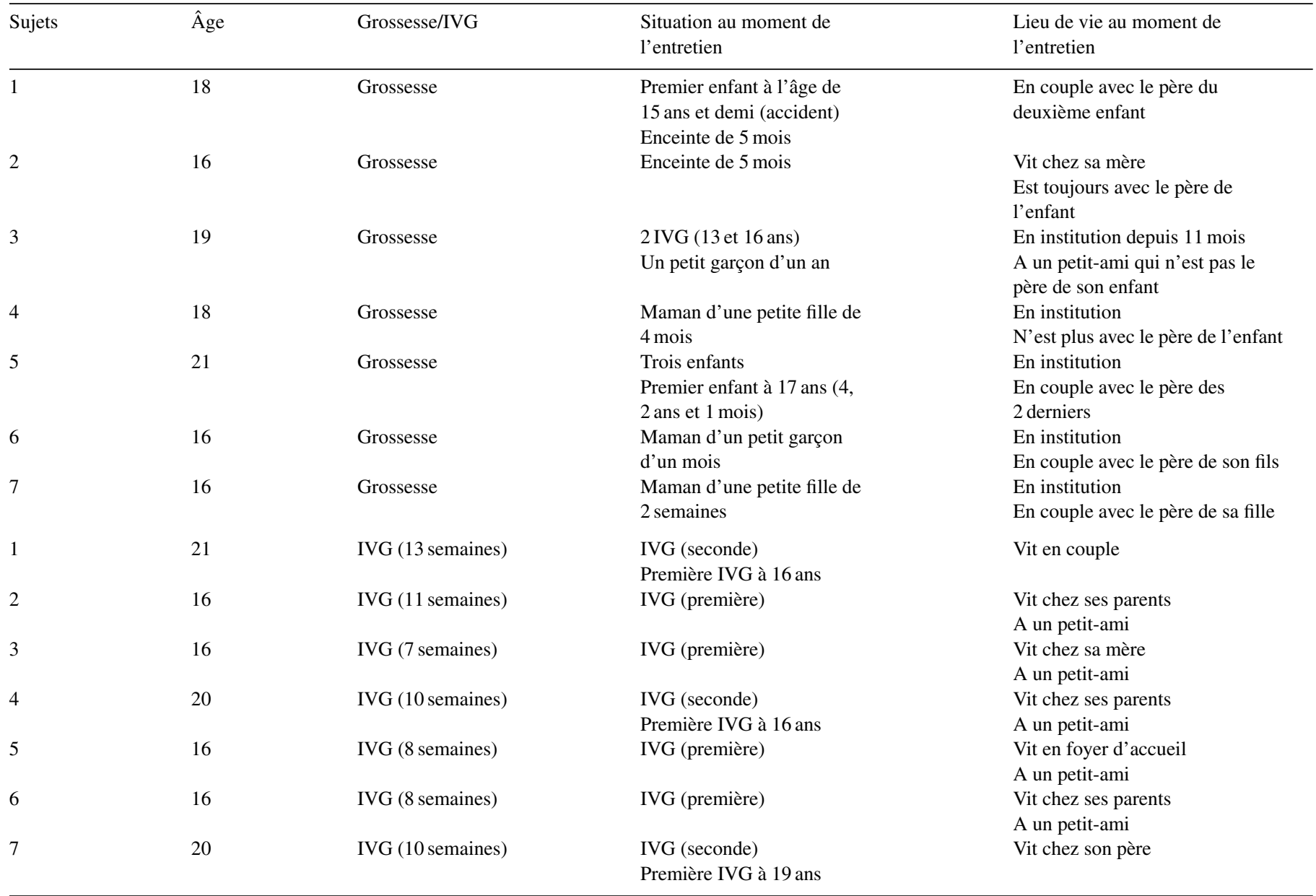

Moyenne d'âge groupe grossesse : 17,7 $\pm 1,9$ ans ; moyenne d'âge groupe IVG : 17,9 $\pm 2,6$ ans.

et une du groupe IVG. L'évaluation a eu lieu après la décision de mener la grossesse à terme ou après l' accouchement, et après l'interruption volontaire de grossesse.

Le groupe témoin était composé de 18 jeunes filles n'ayant jamais fait l'expérience d'une grossesse (moyenne d'âge de 18 ans et demi). Ces jeunes filles ont été recrutées dans différentes écoles secondaires. Au départ, 42 jeunes filles avaient rempli les questionnaires mais uniquement 18 jeunes filles ont été retenues du fait des erreurs de complétion. Des testings ont été proposés à toutes les adolescentes. Plusieurs dimensions ont été évaluées comme la personnalité (impulsivité, l'estime de soi), le support social, le statut socio-économique et les expériences négatives vécues dans l'enfance. Un consentement éclairé a été signé par chacune des participantes.

\subsection{Instruments}

Un entretien semi-structuré en deux volets a été proposé aux groupes IVG et grossesse (les entretiens ont été enregistrés et puis retranscrits). Une première partie, commune aux deux groupes, comprenait des renseignements démographiques, culturels et sociaux (parcours scolaire de la jeune fille, des parents, de la fratrie, lieu de vie, ...). Une deuxième partie plus spécifique à la décision d'IVG ou au maintien de grossesse portait sur les relations affectives, amicales et familiales en vue d'appréhender l'influence des pairs ou de la famille dans la prise de décision. Des informations sur l'âge des premières règles, des premiers rapports sexuels, l'utilisation des moyens de contraception et du type de contraception utilisé ont également été demandées.

Outre l'entretien semi-structuré, quatre tests et échelles structurés ont été administrés pour approcher les dimensions psychologiques.

Le TCI-R junior de Cloninger [19] a été choisi pour évaluer la personnalité des jeunes filles. Il évalue 4 tempéraments qui auraient plutôt une base biologique (recherche de nouveauté, évitement du danger, dépendance à la récompense et persistance) et 3 caractères lesquels seraient plutôt issus des apprentissages sociaux (auto-détermination, coopération et transcendance). Ce test s'adapte particulièrement bien à notre étude car il permet d'évaluer différents facteurs psychologiques reconnus comme susceptibles d'influencer les comportements des adolescents, notamment le goût du risque, l'insouciance et l'immaturité [8]. La recherche de nouveauté et l'évitement du 
danger sont effectivement deux dimensions qui peuvent avoir un impact sur la prise de décision chez les adolescents [20].

L'UPPS Impulsive Behavior Scale mesure l'impulsivité pouvant être définie comme une perte de contrôle qui s'observe au travers d'une large palette de comportements mal conçus, exprimés de manière prématurée, inutilement risqués, ou non appropriés à la situation. Ce test évalue l'urgence, le manque de préméditation, le manque de persévérance et la recherche de sensation. L'UPPS a été traduit en français et validé auprès d'adultes et d'adolescents [21]. Charnigo et al. ont, effectivement, démontré que la recherche de sensation et l'impulsivité dans la prise de décision chez des jeunes adultes étaient positivement corrélées et associées à des comportements sexuels à risque [22].

L'Inventaire de l'Estime de Soi de Coopersmith en forme scolaire mesure l'estime de soi [23] il comporte 58 items, décrivant des sentiments, des opinions ou des réactions d'ordre individuel face auxquels la personne doit se prononcer en cochant « Me ressemble » ou « Ne me ressemble pas ». Les attitudes évaluatives, mesurées par le SEI de S. Coopersmith, couvrent quatre domaines : social, professionnel ou scolaire, familial et général. Il permet d'apprécier dans quel domaine et, dans quelle mesure, les sujets ont une image positive d'eux-mêmes. C'est une mesure directe dont la fidélité et la validité ont été testées sur des enfants français de 12 à 24 ans.

L'Adverse Childhood Experiences (ACE) - Hillis et al. [7] est un questionnaire proposé aux sujets pour évaluer les expériences négatives vécues durant l'enfance. Il est composé de dix questions, chacune renvoyant à une forme de violence, d'abus ou de négligence, d'événements de vie traumatiques ou d'un climat familial délétère. Ce questionnaire semble très largement utilisé dans la littérature anglo-saxonne. Une version de base disponible en français a été utilisée pour cette recherche.

Dans le cadre de notre étude, la procédure de Kruskal-Wallis a été préférée à celle de l'Anova simple classique pour la comparaison des 3 groupes. Le test de Mann-Whitney a été réalisé sur chaque paire de groupe (témoin versus grossesse, témoin versus IVG et enfin grossesse versus IVG), comme post-hoc.

\section{Résultats}

Les trois sous-groupes (grossesse/IVG/témoin) ont été évalués et comparés au niveau quantitatif et qualitatif. Une comparaison statistique entre les groupes, groupe grossesse/IVG et groupe témoin a porté sur le statut économique, le parcours scolaire, la ménarche, le coïtarche, les questions autour de la sexualité, la personnalité, l'impulsivité, l'estime de soi ainsi que les expériences négatives vécues dans l'enfance par les sujets. L'analyse qualitative et clinique de l'étude menée à partir des entretiens semi-structurés a, quant à elle, permis d'étayer les résultats de ces analyses.

\subsection{Statut socio-économique}

Les familles des adolescentes du groupe grossesse sont celles qui ont le statut socio-économique le plus bas, dont les parents, dans tous les cas séparés, vivent de revenus de remplacement (chômage, invalidité, CPAS). De plus, ces adolescentes vivent le plus souvent en institution (Tableau 2). Dans la plupart des familles, les parents n'ont aucune qualification et n'ont pas fini leurs études secondaires. Ces jeunes filles proviennent de milieux socio-économiques précaires, de familles monoparentales et bénéficiant d'allocations de remplacement.

\subsection{Support social}

Au niveau du support social, on relève une différence significative entre le groupe témoin et le groupe grossesse en termes de nombre d'amis et en termes de support social. Les jeunes filles ayant choisi de garder leur enfant ont un support social médiocre et moins important en nombre. Le groupe IVG apparaît cependant plus contrasté. L'analyse des entretiens montre, en effet, que le réseau social est souvent plus restreint chez les jeunes filles du groupe grossesse.

\subsection{Parcours scolaire}

Les jeunes filles du groupe grossesse ont rencontré plus de difficultés dans leur parcours scolaire : niveau atteint, notes plus basses, plus de redoublements (Tableau 3). Dans le groupe grossesse, 4 adolescentes sur 7 sont en décrochage scolaire. Toutes les jeunes filles du groupe IVG continuent leurs études, travaillent ou sont à la recherche d'un emploi.

\subsection{Facteurs individuels}

\subsubsection{Facteurs biologiques (ménarche, sexualité, contraception, timing pubertaire)}

La ménarche moyenne se situe à $12,5 \pm 1,7$ ans. Il n'y a pas de différence statistiquement significative entre les groupes à ce niveau. Concernant les relations sexuelles, seuls 12 sujets du groupe témoin en ont déjà eues. Chez celles-ci, le coïtarche est plus tardif, aux alentours d'une bonne année et ce, de manière statistiquement significative. Aucune autre différence n'est observée sur les autres variables comme l'âge de la première relation sentimentale, le différentiel ménarche-coïtarche, la durée de la pratique sexuelle, la latence entre le début de la relation amoureuse et coïtarche, le nombre de partenaires sexuels et la durée moyenne des relations sentimentales par partenaire, l'âge du sujet mis en covariance.

\subsubsection{Facteurs de personnalité (impulsivité, estime de soi)}

Trois instruments ont permis d'approcher les facteurs psychologiques des sujets des trois sous-groupes : le TCIJ, l'UPPS et l'inventaire d'estime de soi de Coopersmith. Pour le TCIJ, aucune différence statistiquement significative n'est observée entre les sujets.

À l'UPPS, seule la sous-échelle «manque de préméditation » permet de discriminer les groupes. Le groupe grossesse se différence à la fois du groupe témoin et du groupe IVG en obtenant des valeurs moyennes plus basses.

À l'Inventaire d'Estime de Soi, seul le domaine social montre des différences entre les groupes témoin versus expérimental. Une tendance existe entre les trois groupes. Le groupe grossesse 
Tableau 2

Statut socio-économique.

\begin{tabular}{lccc}
\hline & Témoin $(n=18)(\%)$ & Grossesse $(n=7)(\%)$ & IVG $(n=7)(\%)$ \\
\hline Statut socio-économique & & & 0 \\
$\quad$ Bon & 37,7 & 57,1 & 14,3 \\
Satisfaisant & 62,3 & 42,9 & 28,6 \\
$\quad$ Bas & 0 & & 57,1 \\
Situation professionnelle des parents & & 42,9 & 14,3 \\
$\quad$ Deux parents travaillent & 100 & 14,3 & 28,6 \\
$\quad$ Un parent travaille & 0 & 42,9 & 57,1 \\
Aucun des deux ne travaille & 0 & & 57,1 \\
Type de professions des parents & & 42,9 & 28,6 \\
$\quad$ Allocataires sociaux & 0 & 57,1 & 14,3 \\
Employés/ouvriers & 72,2 & 0 & \\
Cadres & 27,8 & & \\
\hline
\end{tabular}

Tableau 3

Niveau de formation atteint ou en cours.

\begin{tabular}{|c|c|c|c|c|c|}
\hline & \multicolumn{4}{|c|}{ Niveau de formation atteint ou en cours } & \multirow[b]{2}{*}{ Total $(\%)$} \\
\hline & Primaire $(\%)$ & Secondaire professionnel (\%) & Secondaire général (\%) & Supérieur/universitaire (\%) & \\
\hline \multicolumn{6}{|l|}{3 groupes } \\
\hline Grossesse & 28,6 & 57,1 & & 14,3 & 100,0 \\
\hline IVG & & 71,4 & 28,6 & & 100,0 \\
\hline Total & 12,5 & 34,4 & 18,8 & 34,4 & 100,0 \\
\hline
\end{tabular}

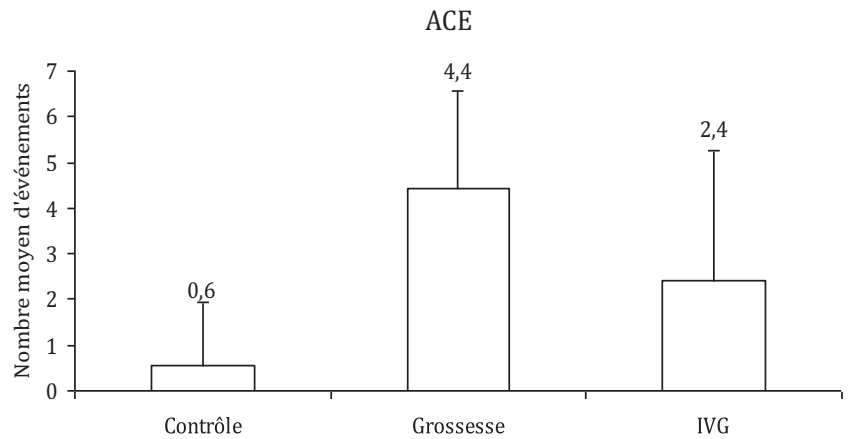

Fig. 1. Adverse Childhood Experiences (nombre moyen d'évènements traumatiques).

est celui qui présente un score moyen en-deçà des valeurs des autres groupes.

\subsection{Expériences traumatisantes vécues dans l'enfance}

L'Adverse Childhood Experiences montre des grandes différences entre les 3 groupes. Chaque question dérivée de l'ACE renvoie à une forme de violence, d'abus ou de négligence, d'événements de vie traumatiques ou d'un climat familial délétère. Les situations de violence psychologique, physique ou sexuelle, de négligence/maltraitance affective ainsi que des situations de rupture familiale sont très présentes dans le groupe grossesse et le groupe IVG, dans près de la moitié des cas (Fig. 1). Pour chaque type de violence, d'abus, de négligence, de maltraitance, il existe des différences statistiquement significatives entre les deux groupes (Tableau 4). De manière générale, c'est dans l'échantillon des adolescentes du groupe grossesse que ces événements traumatisants sont apparus avec la plus grande fréquence; deux fois plus fréquemment que dans le groupe IVG pour les violences physiques et psychologiques et les maltraitances affectives. Une adolescente sur deux de ce groupe a subi une violence sexuelle. En effet, les jeunes filles de ce groupe semblent avoir vécu plus d'évènements traumatisants que celles du groupe témoin, et cette différence est d'autant plus marquée dans le groupe grossesse. L'analyse des trajectoires de vie nous a permis d'étayer notre recherche. Celle-ci a révélé que 11 adolescentes sur 14 ont connu un parcours de vie marqué par plusieurs évènements de vie traumatiques : divorce des parents, des violences conjugales, des placements institutionnels et des abandons.

\section{Discussion}

Bien que l'on parle de grossesse à un âge précoce, il apparait qu'il n'existe pas de relation entre puberté précoce (ménarche avant 12 ans) et grossesse à l'adolescence. En effet, aucun lien entre l'âge de la ménarche et une grossesse à l'adolescence n'a été relevé dans notre échantillon.

Confirmant les études antérieures, notre étude a montré que les jeunes filles ayant vécu une grossesse avaient des connaissances erronées et une mauvaise utilisation de la contraception, comparativement au groupe témoin. Les fausses croyances concernant l'utilisation de la contraception seraient clairement un facteur pouvant expliquer la survenue d'une 
Tableau 4

Adverse Childhood Experiences (résultats).

\begin{tabular}{llll}
\hline Kruskal-Wallis & $(p<0,05)$ & Mann-Whitney $U$ & $(p<0,05)$ \\
\hline Ctrl/GR/IVG & $\mathbf{0 , 0 0 1}$ & Ctrl/GR & $\mathbf{0 , 0 0 0}$ \\
& & Ctrl/IVG & G,097 \\
& & Gr/IVG & 0,165
\end{tabular}

\begin{tabular}{|c|c|c|c|c|c|c|}
\hline Types de violence & Ctrl & Expé & GR & IVG & TOT & $p<0,05$ \\
\hline Violence psychologique familiale & 16,7 & 64,3 & 85,7 & 42,9 & 37,5 & 0,006 \\
\hline Violence physique familiale & 11,1 & 42,9 & 57,1 & 28,6 & 25,0 & 0,040 \\
\hline Violence/abus sexuel & 0,0 & 42,9 & 42,9 & 42,9 & 18,8 & $\mathbf{0 , 0 0 2}$ \\
\hline Négligence/maltraitance affective familiale & 5,6 & 42,9 & 57,1 & 28,6 & 21,9 & 0,011 \\
\hline Négligence/maltraitance éducative & 0,0 & 14,3 & 14,3 & 14,3 & 6,3 & 0,098 \\
\hline Perte/rupture/abandon parental & 0,0 & 50,0 & 57,1 & 42,9 & 21,9 & 0,001 \\
\hline Présence de violences conjugales sur la personne de la mère ou la belle-mère & 5,6 & 21,4 & 28,6 & 14,3 & 12,5 & 0,178 \\
\hline Présence d'un vécu d'une personne souffrant d'un abus de substance(s) & 5,6 & 28,6 & 57,1 & 0,0 & 15,6 & 0,075 \\
\hline Présence d'un vécu d'une personne atteinte de maladie mentale & 5,6 & 28,6 & 42,9 & 14,3 & 15,6 & 0,075 \\
\hline Présence d'un vécu de personne emprisonnée dans la famille & 5,6 & 7,1 & 0,0 & 14,3 & 6,3 & 0,854 \\
\hline ACE Score & 5,6 & 34,3 & 44,3 & 24,3 & 18,1 & \\
\hline
\end{tabular}

IVG : interruption volontaire de grossesse; Ctrl : contrôle; Exp : expérimental; GR : grossesse; TOT: total. Les éléments en gras du tableau correspondent aux valeurs statistiquement significatives (probabilité de dépassement $<0,05$ ).

grossesse. En effet, la plupart des jeunes filles disent avoir eu des informations sur les différents moyens disponibles mais pas concernant leur utilisation. Nous avons relevé une forme de désengagement parental ou d'évitement en ce qui concerne la sexualité de ces adolescentes, la plupart qualifiant leurs parents de «fermés aux conversations portant sur le sexe » et ne pouvant pas s'adresser à eux pour trouver l'information souhaitée. De plus, ces adolescentes, plus isolées socialement, ne peuvent non plus s'appuyer sur des échanges avec des amies pour trouver des informations afin de compenser le manque de communication au sein de leurs familles. Des campagnes de prévention devraient répondre à ces besoins d'informations, dans tous les milieux, notamment sous forme de séances d'éducation à la vie sexuelle et affective dans les écoles. Des animations spécifiques et adaptées à l'âge traitant de la puberté, des relations amoureuses, des conduites à risque, des moyens de contraception devraient être obligatoires dès l'école primaire.

Par ailleurs, les résultats ont mis en lumière que les caractéristiques individuelles ne sont pas un facteur saillant explicatif des grossesses à l'adolescence. La question était de savoir s'il existait un lien entre le fait d'avoir vécu une grossesse à l'adolescence et des scores plus élevés aux dimensions «recherche de nouveauté » ou «évitement du danger» du TCIJ par rapport au groupe témoin, ou encore des scores plus hauts aux sous-échelles «manque de préméditation», «urgence» et «recherche de sensation» à l'UPPS. Les résultats au TCIJ ne montrent aucune différence entre les sujets. À l'échelle l'UPPS, seule la sous-échelle «manque de préméditation» permet de discriminer les groupes. En effet, contrairement à ce que l'on pouvait imaginer, le groupe des jeunes filles qui ont décidé de garder leur enfant se différencie à la fois de celui des jeunes filles qui ont décidé d'interrompre leur grossesse et du groupe témoin, obtenant des valeurs moyennes plus basses sur cette dimension qu'est le « manque de préméditation ». Ce résultat traduit une meilleure capacité des jeunes filles à prendre en compte les conséquences négatives et positives d'une action; si l'on considère cette dimension par rapport au choix de poursuivre la grossesse, il pourrait indiquer que la grossesse pourrait s'inscrire dans un projet de parentalité dans lequel la jeune fille se projette avec des attentes particulières. Les récits des adolescentes montrent que, bien que la grossesse n'ait pas été consciemment désirée ou planifiée au départ, souvent elle ne résulte pas d'un accident de contraception. Cette grossesse surviendrait à un moment où la jeune fille serait en réel questionnement sur sa vie et sur son avenir, et viendrait répondre à un besoin d'émancipation, de réparation et d'accession à un statut plus valorisant.

Les résultats du TCIJ montrent l'absence de différence significative au niveau de la personnalité des jeunes filles du groupe grossesse/IVG par rapport à celles du groupe témoin. Ceci va à l'encontre des études mettant en lien l'impulsivité et la recherche de sensations avec un comportement sexuel à risque et d'une grossesse à l'adolescence [22]. Les facteurs individuels ne seraient donc pas un facteur saillant intervenant dans la prise de décision.

Notre étude s'est intéressée aux facteurs environnementaux et sociaux qui ont une influence sur les conduites des adolescents. Les jeunes filles de notre échantillon qualifient leur milieu socioéconomique de précaire, en comparaison au groupe témoin. Elles sont souvent issues de familles monoparentales et bénéficient de revenus de remplacement. Comme l'a relevé Arai, la grossesse arrive plus fréquemment chez les jeunes filles ayant peu de perspectives scolaires et professionnelles [17]. La maternité représenterait, pour certaines, une manière d'accéder à une position sociale plus valorisante et à un projet de vie. Il s'agit pour ces dernières d'affirmer leur indépendance, de trouver une identité vis-à-vis de leur famille et leurs pairs, d'atteindre un certain statut social (mère) et d'en obtenir les bénéfices secondaires (sociaux et économiques), surtout en cas de grande précarité sociale.

Par ailleurs, il est connu que le rôle des pairs est prépondérant dans le fonctionnement des adolescents, et doit être questionné 
dans toute thématique adolescentaire. Notre étude met en avant que les jeunes filles poursuivant leur grossesse, comparativement aux jeunes filles qui l'interrompent, ne bénéficient pas d'un réseau social contenant et structurant (support social de moindre qualité et cercle d'amis plus restreint). Le manque de support social apparaît comme un des facteurs associé au choix de la poursuite de la grossesse. Ces jeunes filles disposant de faibles ressources sociales et peu de support par les pairs, sont privées d'un lien d'échange informationnel et émotionnel, leur permettant d'envisager la possibilité d'une interruption de grossesse. En outre, on peut également considérer que la grossesse, pour ces jeunes filles, aurait la fonction de compenser le manque de relations sociales et l'isolement social.

À la question de savoir quels sont les facteurs qui sont les plus prégnants dans la poursuite ou non d'une grossesse, il a été très intéressant de constater que les caractéristiques individuelles (psychologiques et biologiques) auraient moins d'impact que les caractéristiques environnementales. Selon Susan Hillis et al. [6,7], un cadre familial soutenant et structurant durant l'enfance protège de l'activité sexuelle précoce, d'une grossesse et de ses conséquences psychosociales négatives à long terme. Ces auteurs ont également mis en avant que les expériences négatives vécues dans l'enfance augmentent le risque d'avoir des comportements sexuels à risque et de connaître une grossesse à l'adolescence. Le rôle du contexte familial est prépondérant dans la construction de la résilience et dans la protection contre les conduites à risque à l'adolescence comme les rapports sexuels précoces, les grossesses, les maladies sexuellement transmissibles et les morts fœtales [7,24]. Effectivement, le contexte familial des adolescentes du groupe grossesse a été souvent marqué par des carences affectives précoces et des traumatismes de toutes sortes (décrochage scolaire, placements multiples, viols, etc.). Le récit des jeunes filles nous renseigne qu'elles ont toutes vécu une séparation parentale ou la perte d'un parent par abandon ou par décès. Cinq adolescentes du groupe grossesse et deux du groupe IVG ont subi des violences physiques et psychologiques graves, de la maltraitance parentale et de nombreux placements. Les résultats au questionnaire ACE confirment statistiquement cette différence significative entre les groupes. Les situations de violence psychologique, physique ou sexuelle, de négligence/maltraitance affective ainsi que des situations de rupture familiale sont très présentes dans le groupe des adolescentes du groupe grossesse/IVG. De manière générale, c'est dans l'échantillon des adolescentes du groupe grossesse que des événements traumatisants sont apparus avec la plus grande fréquence. Il est à noter qu'il existe également un lien entre les carences affectives vécues dans l'enfance et une sexualité précoce où les jeunes filles chercheraient dans l'acte sexuel un échange d'amour dont elles ont manqué dans l'enfance.

Il est clair que l'on aurait tendance à conclure que les violences subies durant l'enfance et l'adolescence et le climat délétère au niveau familial interviendraient de manière spécifique. La poursuite de la grossesse pourrait être sous-tendue par des attentes implicites de réparation alors que pour les IVG, la décision de l'arrêt de grossesse ne serait pas entravée par ces besoins de compensation. Cependant, il pourrait être intéressant de voir en quoi ces traumatismes interviendraient dans la poursuite d'une grossesse de manière spécifique, peut-être en essayant de maîtriser les autres variables et en sélectionnant un échantillon plus important. D'autre part, investiguer la place du père adolescent serait également une piste dans de futures recherches, celui-ci occupant une place prépondérante parmi les facteurs explicatifs du choix de la poursuite de la grossesse.

Malgré l'échantillon restreint, cette étude est l'une des rares à avoir intégré une approche clinique et empirique dans l'étude du choix de poursuivre ou d'interrompre une grossesse à l'adolescence. Elle recouvre des dimensions individuelles et environnementales permettant de mettre en lumière les facteurs de risques psychosociaux liés à une grossesse à l'adolescence. Elle permet de mieux saisir le sens et les enjeux et elle met surtout en avant l'importance d'une intervention précoce.

Une des limites de cette étude est la petite taille et le biais de sélection de l'échantillon. En effet, la difficulté de recrutement nous a contraints à y intégrer 4 jeunes filles vivant en institution, ce qui a été, à la fois intéressant pour l'étude, à la fois limitatif.

La nature même de notre population nous a, cependant, permis de souligner combien les fragilités traumatiques, les vulnérabilités sociales, économiques, relationnelles et familiales, sont, bien plus que les dimensions psychologiques de la personnalité, des facteurs qui sous-tendent la survenue de la grossesse et la poursuite d'un projet de parentalité. Des études à partir d'échantillons plus larges et diversifiés devraient se poursuivre dans une perspective bio-psycho-sociale intégrant toutes ces variables.

\section{Implications}

Les programmes de prévention doivent, à notre sens, être davantage investis par les politiques de santé publique, sous forme d'informations d'éducation à la sexualité et ce, dès l'enseignement primaire. Toutefois, ces actions doivent dépasser l'information sur la contraception et son utilisation, car la grossesse à l'adolescence est bien plus qu'un défaut ou un échec de contraception, elle répond, pour de nombreuses adolescentes, à des attentes implicites d'une identité nouvelle et de réparation par rapport à des fractures et traumatismes antérieurs. Des programmes de prévention basés sur des activités pédagogiques sous forme de jeux de rôle, de projections vidéo et de groupes de parole abordant ces thèmes devraient être développés au sein des établissements scolaires, et particulièrement ceux drainant des populations plus vulnérabilisées. Ils permettraient également de renforcer les liens entre pairs et de constituer des soutiens sociaux et émotionnels pour ces adolescents.

Plus spécifiquement, un espace de parole ainsi qu'un soutien psychologique devraient être proposés aux jeunes filles confrontées à une grossesse, qui sont souvent isolées et socialement livrées à elles-mêmes au moment difficile de la prise de décision. Un accompagnement à plus long terme après l'IVG devrait être prévu systématiquement pour chaque jeune fille.

Développer dans le cadre scolaire, et surtout dans les établissements scolaires (souvent de type professionnel ou alternatif) qui comptent dans leur population scolaire le plus d'adolescents parents, des programmes spécifiques proposés aux jeunes mères adolescentes, dispensés durant les horaires scolaires, semble 
une mesure préventive majeure. Ils permettraient d'assurer un soutien à la parentalité mais également de contrer le risque d'un décrochage scolaire de ces jeunes mères.

Enfin, eu égard aux difficultés psychosociales et au vécu d'adversité des mères adolescentes qui ne sont plus scolarisées, des interventions spécifiques de soutien à la parentalité, en vue de renforcer leurs compétences et sentiments de compétence parentale, et les sensibiliser à leurs propres besoins et à ceux de leur enfant, pourraient être menées à partir d'une clinique en milieu de vie et d'interventions à domicile.

\section{Déclaration d'intérêts}

Les auteurs déclarent ne pas avoir de conflits d'intérêts en relation avec cet article.

\section{Références}

[1] Organisation mondiale de la santé. Grossesse à l'adolescence, un problème culturel complexe. Bulletin de l'Organisation mondiale de la santé 2009;87:405-84 [Available from: URL: http://www.who.int/bulletin/ volumes/87/6/09-020609/fr/index.html].

[2] Organisation mondiale de la santé. Mariages précoces, grossesses chez les adolescentes et les jeunes femmes. Soixante-cinquième Assemblée Mondiale de la Santé. Point 13.4 de l'ordre du jour provisoire; 2012.

[3] Organisation mondiale de la santé. Statistiques sanitaires mondiales. Genève: OMS; 2012

[4] Moreau N, Swennen B, Roynet D. Étude du parcours contraceptif des adolescentes confrontées à une grossesse non prévue: éléments pour une meilleure prévention? Bruxelles: Gacehpa ASBL et le Centre d'Études et de Recherche en Santé Publique (CERESP); 2006.

[5] Ribera L, Pérez G, Salvador J, Borrell C. Socioeconomic inequalities in unintended pregnancy and abortion decision. J Urban Health 2007;85:1, http://dx.doi.org/10.1007/s11524-007-9233-z.

[6] Hillis SD, Anda RF, Dube SR, Felitti VJ, Marchbanks PA, Macaluso M. The protective effect of family strengths in childhood against adolescent pregnancy and its long-term psychosocial consequences. Perm J 2010;14:18-27.

[7] Hillis SD, Anda RF, Dube SR, Felitti VJ, Marchbanks PA, Marks JS. The association between adverse childhood experiences and adolescent pregnancy, long-term psychosocial consequences, and fetal death. Pediatrics 2004;113:320-7

[8] Miller BC, Benson B, Galbraith KA. Family relationship and adolescent pregnancy risk: a research synthesis. Dev Rev 2001;21(1):1-38.

[9] Le Van C. Les grossesses à l'adolescence : normes sociales, réalités vécues. Paris: L'Harmattan; 1998.
[10] Loignon C. Prévention et soutien de la grossesse et de la maternité/paternité à l'adolescence. État de la situation pour la région de Montréal. Rapport de recherche mené dans le cadre du programme projet placement carrière; 1996.

[11] Daroorian D. Grossesses adolescentes. La vie de l'enfant. Toulouse: Érès; 2007.

[12] Berrewaerts J, Noirhomme-Renard F. Les grossesses à l'adolescence : quels sont les facteurs explicatifs identifiés dans la littérature? (Dossier technique 06-40). Louvain-La-Neuve, Belgique: UCL-RESO. Unité d'éducation pour la santé. École de santé publique, faculté de médecine; 2006.

[13] Faucher P, Dappe S, Madelenat P. Maternité à l'adolescence : analyse obstétricale et revue de l'influence des facteurs culturels, socio-économiques et psychologiques à partir d'une étude rétrospective de 62 dossiers. Gynecol Obstet Fertil 2002;30:944-52.

[14] Wendland J, Levandowski DC. Les pères adolescents: le versant oublié des grossesses à l'adolescence. Neuropsychiatr Enfance Adolesc 2011;59:433-8.

[15] Corcoran J, Franklin C, Bennett P. Ecological factors associated with adolescent pregnancy and parenting. Soc Work Res 2000;24:29-39.

[16] Aujoulat I, Libion F, Rose B. Les grossesses chez les adolescentes: analyse des facteurs intervenant dans la survenue et leur issue, Vol.2. Unité d'éducation pour la santé RESO. Université catholique de Louvain; 2007.

[17] Arai L. Low expectations, sexual attitudes and knowledge: explaining teenage pregnancy and fertility in English communities, Insights from qualitative research. Londres: The Editorial Board of The Sociological Review; 2003. p. 119-216.

[18] Bérard A-M. Désirer ou avoir un enfant à l'adolescence : mieux saisir le sens et les enjeux. Ça s'exprime. Ministère de la Santé et des Services Sociaux du Québec. Université du Québec; 2007.

[19] Hansenne M. Le modèle biosocial de la personnalité de Cloninger. L'année psychologique 2001;101(1):155-81, http://dx.doi.org/10.3406/ psy.2001.29720.

[20] Whiteside SP, Lynam DR. The five factor model and impulsivity: using a structural model of personality to understand impulsivity. Pers Individual Differences 2001;30:669-89.

[21] Van Der Linden M, d'Acremont M, Zermatten A, Jermann F, Laroi F, Willems S, et al. A French Adaptation of the UPPS Impulsive Behavior Scale: confirmatory factor analysis in a sample of undergraduate students. Eur J Psychol Assess 2006;22:38-42.

[22] Charnigo R, Noar SM, Garnett C, Crosby R, Palmgreen P, Zimmerman RS. Sensation seeking and impulsivity: combined associations with risky sexual behavior in a large sample of young adults. J Sex Res 2013;50(5): 480-8.

[23] Coopersmith S. Manuel d'inventaire d'estime de soi-SEI Forme Scolaire. Paris: Les Éditions du Centre de Psychologie Appliquée; 1984.

[24] Dietz PM, Spitz AM, Anda RF, Williamson DF, McMahon PM, Santelli JS. Unintended pregnancy among adult women exposed to abuse or household dysfunction during their childhood. JAMA 1999;282:1359-64. 